\title{
Synthesis and Fluorescence Behavior of Cation Chemosensor Based on Azacrown Ether Fluoroionophore Carrying Styrylanthracene
}

\author{
Eun Ju Shin \\ Department of Chemistry, Sunchon National University, Sinchon, Chomam 540-742, Korea. E-mail: ejs@sunchon.ac.kr \\ Received September 8,2006
}

Key Words : Azacrown ether, Chemosensor, Styrylanthracene, Fluoroionophore

Recently, the development of chemosensor associated with fluoroionophore has attracted great attention. Fluoroionophore ${ }^{1-9}$ is a compound combining the ionophore and fluorophore, where ionophore binds selectively with a specific ion and fluorophore generates a fluorescence signal upon ion binding.

Depending on the nature of fluorophore-ionophore interaction in fluoroionophore, fluorescence properties of fluorophore such as fluorescence intensity or fluorescence wavelength maximum may be influenced by ion capture of ionophore..$^{1-9}$ Sensitivity of fluorophore may be associated with the ionic recognition ability of the ionophore, if the fluorophore may be perturbed by the coordination of cation. The recent increase of interest in the synthesis of crown ether derivatives incorporating different fluoroionophores is recently due to their application as alkali metal ion sensors in biochemical analysis and medical diagnostics.

In particular, azacrown ether, a typical ionophore, acts as an electron-rich donor as well as a ligand of the cation. The electron pair on the nitrogen heteroatom in electron-rich azacrown ether could be transferred towards electron deficient fluorophore in a $\pi$-conjugated D-A arrangement, through the charge transfer interaction between ionophore, an electron donor, and fluorophore, an electron acceptor. ${ }^{10-17}$ The efficient intramolecular charge transfer takes part in the modification of the first electronic transition state of the fluorophore and may lead to weaken the fluorescence from fluorophore. Upon binding of a cation into ionophore cavity, the electron pair on nitrogen heteroatom of ionophore is stabilized and the intramolecular charge transfer is inhibited. Therefore, the original electronic configuration of the unsubstituted fluorophore is recovered, so that fluorescence may be strengthened. Many fluoroionophores including stilbenyl- ${ }^{10-12}$ aryl-, ${ }^{13.14,16}$ or styryl-crown ether ${ }^{15.17}$ derivatives containing a stilbene, styrene or aryl moiety as a fluorophore and a crown ether/azacrown ether moiety as an ionophore have been investigated. However, the main disadvantage of these fluoroionophore is the relatively small changes in fluorescence intensity upon cation binding. Incorporation of more efficient fluorophore such as anthracene into fluoroionophore may lead to more remarkable change of fluorescence signal depending on the cation binding. ${ }^{16}$

Recent work has concemed the binding of various fluorophores to the $N$-phenylaza-15-crown-5 and their spectroscopic and complexation properties. This paper concenns the synthesis of $N$-phenylaza-15-crown-5 derivative containing anthrylethene chromophore ${ }^{18.19}$ and the effect of the complexation with various alkali metal cations on the absorption and fluorescence behavior.

We prepared $N$-[4-(2-anthracen-9-yl-viny])-pheny]]-aza15-crown-5 (1), a azacrown ether derivative containing 1-(9anthryl)-2-phenylethene (9-APE) moiety, by Wittig coupling between $N$-[4-formyl-phenyl]-aza-15-crown-5 and 9bromomethylanthracene.

It is known that $t-9-\mathrm{APE},{ }^{20}$ a styrene derivative containing highly fluorescent anthracene, shows fairly strong fluorescence $\left(\Phi_{i}=0.45, \lambda_{i}^{\max }=476 \mathrm{~nm}\right.$ in acetonitrile), coming from the locally excited state which the excitation energy is localized on anthracene ring. For 1-(9-anthry])-2-(4-methoxyphenyl)ethene (MeO-APE) containing an electron donating substituent methoxy group, ${ }^{20}$ fluorescence becomes weak and is red-shifted $\left(\Phi_{\mathrm{r}}=0.028, \lambda_{i}^{\mathrm{max}}=494 \mathrm{~nm}\right.$ in acetonitrile) due to the contribution of the intramolecular
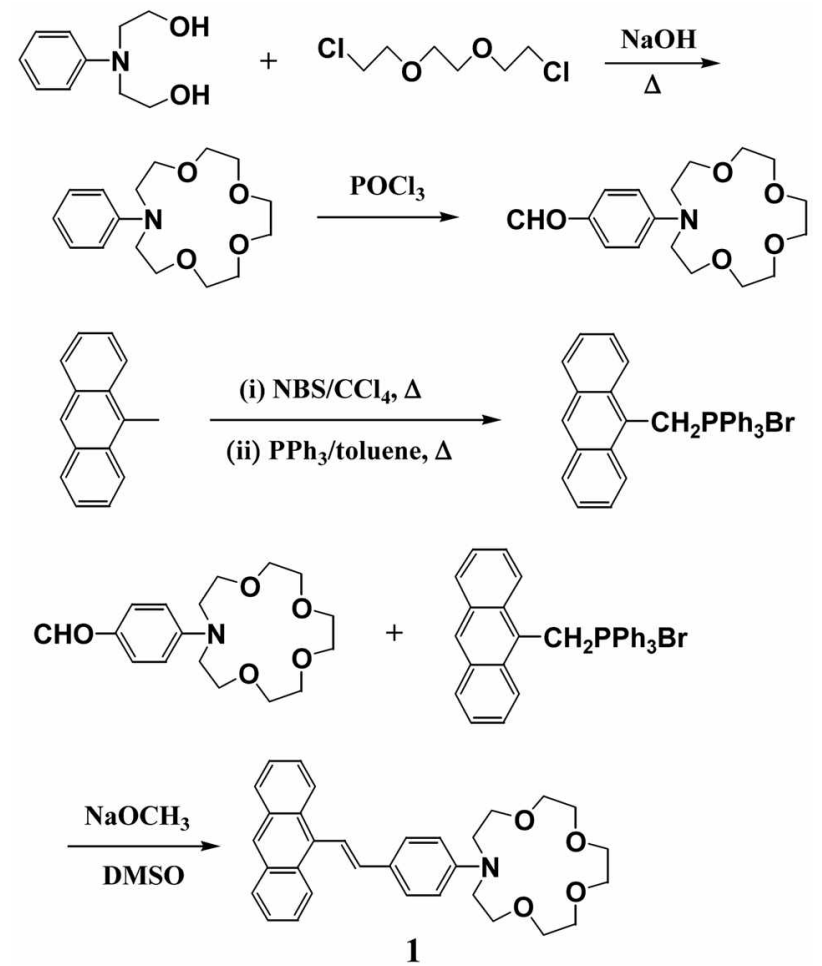

Scheme 1. Synthesis of $N$-[4-(2-anthracen-9-yl-vinyl)-phenyl]-aza$15-\operatorname{crown}-5(\mathbf{1})$. 
charge transfer state to the lowest excited state.

It is also expected that 9-APE derivative containing the electron-rich azacrown ether moiety shows weak fluorescence due to the efficient intramolecular charge transfer. In fact, fluorescence quantum yield of $\mathbf{1}$ was small in acetonitrile $\left(\Phi_{\mathrm{f}}=0.05, \lambda_{\mathrm{i}}^{\mathrm{max}}=500 \mathrm{~nm}\right)$, similar to MeO-APE (see Table 1). As metal cation is added, the cation is inserted into the azacrown cavity of $\mathbf{1}$, in which nonbonding electron pair on nitrogen atom of azacrown ether is stabilized. As a result, intramolecular charge transfer from azacrown ether moiety to anthrylethene moiety is inhibited, so that fluorescence intensity will be restored upto that of the unsubstituted compound, 9-APE.

Figure 1 and Figure 2 show the absorption and fluorescence spectra of 1 with various alkali metal cations in acetonitrile, respectively. The concentrations of 1 and metal perchlorate were $1 \times 10^{-5} \mathrm{M}$ and $\mathrm{I} \times 10^{-2} \mathrm{M}$, respectively. In the absence of metal cation, absorption wavelength maxima of 1 in acetonitrile are 376,385, $413 \mathrm{~nm}$ and fluorescence wavelength maximum is $500 \mathrm{~nm}$. Fluorescence excitation wavelength of $360 \mathrm{~nm}$ was employed. On the addition of metal cations, the absorption maximum and intensity of 1 was nearly unchanged. The fluorescence maximum shifted to short-wavelength and its intensity was increased in the presence of some metal cations. In the presence of $\mathrm{K}^{+}$or $\mathrm{Cs}^{+}$, both absorption and fluorescence spectra was nearly unchanged. As $\mathrm{Na}^{+}$cation is added, fluorescence intensity was slightly increased. With the addition of $\mathrm{Li}^{+}$cation, fluorescence intensity was remarkably increased to around 4 fold with blue-shift of its wavelength. Spectroscopic data and association constants of $\mathbf{1}$ in the presence of various alkali metal cations were presented in Table 1.

The complexation of cation on a fluoroionophore can cause the change in fluorescence yield, critically depending on the change of the relative position of the excited state energy levels involved such as strongly emissive locally excited state and weakly emissive intramolecular charge transfer state. Upon cation addition, a charge transfer from the lone pair on the nitrogen atom of the azacrown ether to the cation may change the intramolecular charge transfer state into the locally excited state. In other words, the reduction of the electron-donating character of the azacrown ether group toward the APE moiety leads to a decrease of the charge transfer to the entire conjugated system, judging from high fluorescence enhancement of 1. Cation complexation to 1 increases the formation of emissive locally excited state through the inhibition of intramolecular charge transfer

Table 1. Spectroscopic data and association constants for $\mathbf{l}$ with various alkali inctal perchlorates in acetonitrile

\begin{tabular}{|c|c|c|c|c|c|}
\hline Salt & $\lambda^{n+1 / 40 m}$ & $\lambda_{t}^{*} / \mathrm{nm}$ & $\Phi_{\mathrm{t}}$ & $\mathrm{I}_{\mathrm{f}} / \mathrm{I}_{\mathrm{f}}^{0}$ & $\mathrm{~K}_{\mathrm{f}}$ \\
\hline none & $376,385,413$ & 500 & 0.05 & 1.0 & - \\
\hline $\mathrm{LiClO}_{4}$ & $376,385,413$ & 480 & - & 3.8 & 10,200 \\
\hline $\mathrm{NaClO}_{4}$ & $376,385,413$ & 482 & - & 1.6 & 340 \\
\hline $\mathrm{KClO}_{4}$ & $376,385,413$ & 500 & - & 1.0 & -0 \\
\hline $\mathrm{CsClO}_{4}$ & $376,385,413$ & 500 & - & 1.0 & -0 \\
\hline
\end{tabular}

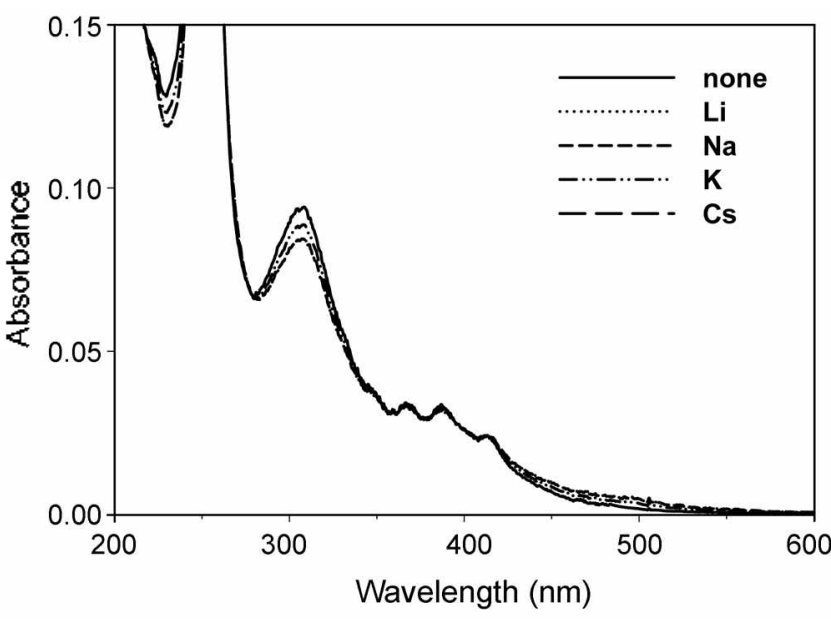

Figure 1. The absorption spectra of 1 in acctonitrile with various alkali inetal perchlorates (none: solid, $\mathrm{LiClO}_{4}$ : dotted, $\mathrm{NaClO}_{4}$ : short-dashed, $\mathrm{KClO}_{4}$ : dash-dot-dotted, $\mathrm{CsClO}_{4}$ : medium-dashed line).

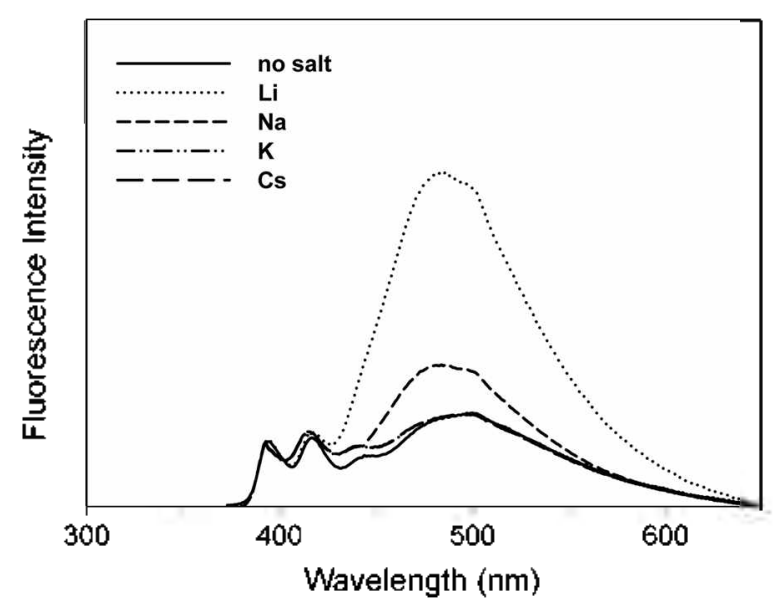

Figure 2. The fluorescence spectra of 1 in acctonitrile with various alkali inetal perchlorates (none: solid, $\mathrm{LiClO}_{4}$ : dotted, $\mathrm{NaClO}_{4}$ : short-dashed, $\mathrm{KClO}_{4}$ : dash-dot-dotted, $\mathrm{CsClO}_{4}$ : long-dashed line).

and leads to the enhanced fluorescence. Fluorescence enhancement by the addition of alkali metal cation was highest for $\mathrm{Li}^{+}$among the alkali metal cations.

In summary, we have prepared a fluoroionophore, $N$ - $[4-$ (2-anthracen-9-yl-vinyl)-phenyl]-aza-15-crown-5. The efficient coordination of $\mathrm{Li}^{+}$cation into azacrown ether cavity in 1 should lead to the fluorescence enhancement. The efficient $\mathrm{Li}^{\prime}$ cation-induced enhancement of the fluorescence can be achieved for a simple fluoroionophore 1 and it allows the use of $\mathbf{1}$ as a chemosensor for $\mathrm{Li}^{+}$cation.

\section{Experimental Section}

Synthesis of $N$-[4-(2-anthracen-9-yl-vinyl)-phenyl]aza-15-crown-5 (1). N-[4-(2-anthracen-9-y]-vinyl)-phenyl]aza-15-crown-5 (1) was prepared Wittig coupling between 9-bromomethyl-anthracene ${ }^{1821}$ and $N$-(4-formylphenyl)-aza15-crown-5. 
To $17.8 \mathrm{~g}(0.10 \mathrm{~mol})$ of $\mathrm{N}$-phenyldiethanolamine in 150 $\mathrm{mL}$ of dioxane at room temperature was added $8.6 \mathrm{~g}(0.22$ $\mathrm{mol}$ ) of $\mathrm{NaOH}$. The suspension was heated to $70-80^{\circ} \mathrm{C}$ and then $18.6 \mathrm{~g}(0.13 \mathrm{~mol})$ of 1,2-bis(2-chloroethoxy)ethane was added. The reaction mixture was refluxed for 2 days and evaporated under vacuum. Column chromatography on silica gel with diethyl ether/ethyl acetate $(1 / 2)$ gave $4.3 \mathrm{~g}$ of $N$-phenyl-aza-15-crown-5 in ca. $15 \%$ yield. 'H NMR $\left(\mathrm{CDCl}_{3}, 400 \mathrm{MHz}\right) \delta 3.62-3.82(\mathrm{~m}, 16 \mathrm{H}$, azacrown ether ring), 4.12-4.14 (m, $4 \mathrm{H}$, azacrown ether ring), $6.70(\mathrm{~d}, 3 \mathrm{H}, J$ $=8.2 \mathrm{~Hz}$, benzene), $7.21(\mathrm{t}, 2 \mathrm{H}, J=8.2 \mathrm{~Hz}$, benzene).

$2.95 \mathrm{~g}(0.01 \mathrm{~mol})$ of $N$-phenyl-aza-15-crown- 5 was dissolved in $5 \mathrm{~mL}$ of DMF on heating and then cooled to -10 ${ }^{\circ} \mathrm{C} .1 .5 \mathrm{~g}(0.01 \mathrm{~mol})$ of $\mathrm{POCl}_{3}$ was rapidly added and the mixture was stirred for $10 \mathrm{~min}$. The solution was warmed to room temperature and stirred for $2 \mathrm{hrs}$ and then subsequently stirred for $5 \mathrm{hr}$ at $100^{\circ} \mathrm{C}$. The solution was cooled to room temperature and poured into $50 \mathrm{~g}$ ice and the mixture was stirred for $30 \mathrm{~min}$. $\mathrm{pH}$ of the solution was adjusted to 7 using $40 \%$ aqueous $\mathrm{NaOH}$ solution. The mixture was filtered and dried with $\mathrm{P}_{4} \mathrm{O}_{10}$ and recrystallized with petroleum ether/ ethyl acetate. $2.4 \mathrm{~g}$ of greenish crystalline $\mathrm{N}$-(4-formylphenyl)-aza-15-crown-5 was obtained in $c a .75 \%$ yield. ' $\mathrm{H}$ NMR $\left(\mathrm{CDCl}_{3}, 400 \mathrm{MHz}\right) \delta 3.62-3.82(\mathrm{~m}, 16 \mathrm{H}$, azacrown ether ring), 4.12-4.14 (m, 4H, azacrown ether ring), $6.74(\mathrm{dd}$, $2 \mathrm{H}, J=2.6,11.6 \mathrm{~Hz}$, benzene), 7.75 (dd, $2 \mathrm{H}, J=2.6,11.6$ $\mathrm{Hz}$, benzene), $9.77(\mathrm{~s}, 1 \mathrm{H}$, aldehyde).

To $0.53 \mathrm{~g}(1 \mathrm{mmol})$ of 9-anthrylmethyltriphenylphosphonium bromide ${ }^{19.20}$ in $20 \mathrm{~mL}$ of DMSO was added $65 \mathrm{mg}$ $(1.2 \mathrm{mmol})$ of sodium methoxide. The solution was refluxed for $1 \mathrm{hr}$ and then $0.3 \mathrm{~g}(1 \mathrm{mmol})$ of $N$-(4-formylphenyl)-aza15 -crown- 5 was added. The mixture was refluxed overnight and then cooled to room temperature. The resulting solution was extracted with water and diethyl ether. The organic layer was evaporated and purified with column chromatography on silica gel with diethyl ether/hexane (5/1). $0.12 \mathrm{~g}$ of orange $\mathrm{N}$-[4-(2-anthracen-9-yl-vinyl)-phenyl]-aza-15-crown-5 was obtained in ca. $25 \%$ yield.

Spectral data for 1: ' $\mathrm{H} \mathrm{NMR}\left(\mathrm{CDCl}_{3}, 400 \mathrm{MHz}\right) \delta 3.62-$ $3.82(\mathrm{~m}, 16 \mathrm{H}$, azacrown ether ring), 4.12-4.14 $(\mathrm{m}, 4 \mathrm{H}$, azacrown ether ring), $6.24(\mathrm{~d}, 2 \mathrm{H}, J=9.0 \mathrm{~Hz}$, benzene), 6.70 (d, $2 \mathrm{H}, J=9.0 \mathrm{~Hz}$, benzene), $7.02(\mathrm{~d}, 1 \mathrm{H}, J=16 \mathrm{~Hz}$, ethylene), 7.43-7.48 ( $\mathrm{m}, 5 \mathrm{H}$, anthracene-2,3,6,7 \& ethylene), $8.0(\mathrm{~d}, 2 \mathrm{H}, J=8.4 \mathrm{~Hz}$, anthracene- 1,8$), 8.28-8.30$ (dd, $2 \mathrm{H}, J$ $=0.6$ and $8.5 \mathrm{~Hz}$, anthracene-4,5), $8.45(\mathrm{~s}, 1 \mathrm{H}$, anthracene 10); MS: $\mathrm{m} / \mathrm{z} 497\left(\mathrm{M}^{+}\right)$.

Spectroscopic measurements. ${ }^{1} \mathrm{H}$ NMR spectra were measured on a $400 \mathrm{MHz}$ Bruker Avance 400 NMR spectrometer in chloroform- $\mathrm{d}_{1}$. Mass spectra were measured on Micromass (UK) platform II GC/LC Mass Spectrometer and Jeol LTD JMS-HX110/110A High Resolution Tandem Mass Spectrometer. Absorption spectra were recorded on a Shimadzu UV-2401PC spectrophotometer. Steady-state fluorescence spectra were recorded on a SLM-Aminco AB2 luminescence spectrophotometer. The concentrations of 1 and alkali metal perclorates were used to be $1 \times 10^{-5} \mathrm{M}$ and
$1 \times 10^{-5} \mathrm{M}$, respectively. Fluorescence spectra were corrected for the wavelength response of the system. The formation constants $\mathrm{K}_{1}$ for the complex formation between $N$-[4-(2anthracen-9-yl-vinyl)-phenyl]-aza-15-crown-5 (1) and alkali metal cations were calculated from the change of fluorescence intensity with the concentration of alkali metal cation.

$$
1 /\left(\mathbf{I}_{0}-\mathbf{I}_{\mathbf{X}}\right)=1 /\left(\mathrm{I}_{0}-\mathrm{I}\right)+1 /\left(\mathbf{I}_{0}-\mathbf{I}\right) \times 1 / \mathrm{K}_{\mathbf{f}} \times 1 /[\mathbf{L}]
$$

where, $I_{0}$ is the fluorescence intensity when [alkali metal cation] $=0, I_{x}$ is the fluorescence intensity of the solution with [alkali metal cation] and $\mathrm{I}$ is the fluorescence intensity when the concentration of alkali metal cation is very high, and we have practically only pure coordinated complex.

Acknowledgements. The author would like to thank KBSI for their help in the measurement of mass spectra. This paper was supported by NON-DIRECTED RESEARCH FUND, Sunchon National University.

\section{References}

I. Czarnik, A. W, Fluorescent Chemosensors for Ion and Molecule Recognition, American Chemical Society: Washington, D.C., 1992.

2. Desvergne, J.-P.; Czarnik, A. W. Chemosensors of Ion and Molectle Recognition; Kluwer Academic Publishers: Dordrecht, Netherlands, 1997.

3. Czarnik, A. W. Acc. Chem. Res. 1994, 27, 302.

4. Valeur, B. Topics in Fluorescence Spectroscopy: Probe Design and Chemical Sensing, Lakowicz, J. R, Ed.; Plenum: New York, $1994 ; \mathrm{Vol} 4$.

5. Valeur, B.; Leray, I. Coord. Chem. Rev, 2000, 205,3.

6. de Silva, A. P.; Gunaratne, H. Q. N.; Gunnlaugsson, T.; Huxley, A. J. M.; McCoy, C. P.; Rademacher, J. T.; Rice, T. E. Chem. Rev. $1997,97,1515$.

7. Fabbrizzi, L.; Poggi, A, Chem. Soc, Rev, 1994, 97, 197.

8. Fabbrizzi, L.; Lincchelli, M.; Pallavicini, P. Acc. Chem. Res, 1999, 32,846 .

9. Amendola, V.; Fabbrizzi, L.; Lincchelli, M.; Mangano, C.; Pallavicini, P.; Parodi, L.; Pogoi, A. Coord. Chem. Rev. 1999, 190. 192.646.

10. Lélard, J.-F.; Lapouyade, R,; Retlig, W. Pure Appl. Chem, 1993, 65,1705 .

11. Dumon, P.; Jonusauskas, G.; Dupuy, F.; Pée, P.; Rullière, C.; Létard, J.-F.; Lapouyade, R. J. Phys. Chenı. 1994, 98, 10391.

12. Mathevet, R.; Jonusauskas, G.; Rullière, C.; Létard, J.-F.; Lapouyade, R. J. Phys. Chem. 1995, 99, 15709.

13. Crochet, P.; Malval, J.-P.; Lapouyade, R. Chem. Commun, 2000, 289.

14. Malval, J.-P.; Gosse, I.; Morand, J.-P.; Lapouyade, R. J. Am. Chent. Soc. 2002, 124, 904.

15. Rurack, K.; Rettig. W; Resch-Genger, U. Chem. Conmtim. 2000 , 407.

16. Witulski, B.; Weber, M.; Bergsträsser, U.; Desvergne, J.-P.; Basssani, D. M.; Bouas-Laurent, H. O/g. Lett, 2001, 3, 1467.

17. Cazaux, L.; Fasher, M.; Lopez, A.; Picard, C.; Tisnes, P. J. Photochent. Photobiol. A: Chem. 1994, 77, 217.

18. Shin, E. J. Bull. Korean Chem. Soc. 2004, 25, 907.

19. Shin, E. J. Bull. Korean Chem. Soc. 2006, 27,751.

20. Sun, L.; Gömer, H. J. Phys, Chem, 1993, 97, 11186.

21. Shin, E. J.; Bae, E. Y.; Kim, S. H.; Kang, H. K.; Shim, S. C. J. Photochem. Photobiol. A: Chem. 1997, 107, 137. 